requiring stenting, particularly patients with tandem occlusion. Further prospective studies are warranted to expand its use in neuro-interventions.

Disclosures A. Ouf: None. S. ElSayed: None. N. Musallam: None. N. Davis: None. M. Tanprayoon: None. J. Fifi: None. J. Mocco: None. H. Shoirah: None.

\section{E-047 ACUTE STROKE THROMBECTOMY IN THE POSTERIOR CIRCULATION IN THE UNITED STATES: REAL WORLD EVIDENCE FROM THE NEUROVASCULAR QUALITY INITIATIVE-QUALITY OUTCOMES DATABASE}

${ }^{1} D$ Cantrell, ${ }^{1} R$ Abdalla*, ${ }^{2} A$ Shaibani, ${ }^{2} M$ Hurley, ${ }^{2} B$ Jahromi, ${ }^{2} M$ Potts, ${ }^{3} S$ Ansari, on behalf of the NVQI-QOD registry. ${ }^{1}$ Radiology, Northwestern University - Feinberg school of Medicine, Chicago, IL; ${ }^{2}$ Radiology, Neurosurgery, Northwestern University - Feinberg school of Medicine, Chicago, IL; ${ }^{3}$ Radiology, Neurosurgery and Neurology, Northwestern University - Feinberg school of Medicine, Chicago, IL

\subsection{6/neurintsurg-2020-SNIS.83}

Introduction Endovascular stroke thrombectomy is currently the standard of care for anterior circulation large vessel occlusions (LVO), based on multiple randomized control trials. Due to the grave consequences of posterior circulation occlusions, no randomized control trials have been performed to assess the benefit and outcome of mechanical thrombectomy in the posterior circulation. We aim to evaluate the real-world evidence for mechanical thrombectomy in posterior circulation LVO using the Neurovascular Quality Initiative (NVQI) registry.

Methods Data from the NVQI registry database was analyzed and compared for anterior versus posterior circulation acute ischemic stroke treated with endovascular thrombectomy in 23 US centers (17 states) from February 2015 to October 2019. Mann Whitney and Fischer exact tests were used for continuous and categorical variables, respectively.

Results Posterior circulation (PC) thrombectomy was performed in a minority of 9.2\% (284/3103 patients) compared to anterior circulation (AC) thrombectomy. LVO sites were predominantly basilar artery occlusions (68\% basilar trunk and $19.4 \%$ basilar apex/PCA) with the remainder as vertebral artery occlusions (12.7\%). Mean age at the intervention was $66 \pm 15.5$ years $(61.6 \%$ male) PC versus $68.8 \pm 15.4$ years AC, $\mathrm{p}=0.004$. Mean NIHSS at presentation was $17 \pm 10.2$ PC versus $15.8 \pm 6.8 \mathrm{AC}, \mathrm{p}=0.007$. CT/CTA was performed in $94 \%$ of cases whereas MRI was performed in $19 \%$ of cases (versus $14 \%$ AC, $\mathrm{p}=0.03$ ). Median arrival to groin puncture time was $110 \mathrm{~min}$ PC versus $87 \mathrm{~min} \mathrm{AC}$, onset to groin time was 375 min PC versus $291 \mathrm{~min} \mathrm{AC}$, and median procedure time was $38 \mathrm{~min}$ PC versus $33 \mathrm{~min}$ AC. Successful recanalization was achieved in $84.5 \%$ (TICI $2 \mathrm{~b} / 3$ ) versus $84 \% \mathrm{AC}(\mathrm{p}=0.87)$ with $50.4 \%$ reporting single pass recanalization and $3.9 \%$ technical failures (3.2\% access related). Intraprocedural complications were reported in $4.9 \%$ of cases $(0.7 \%$ vessel perforations, $0.4 \%$ hemorrhagic transformations, $1.4 \%$ non-target embolization, and $1.8 \%$ iatrogenic dissections). Mean post-operative length of stay was $9.5 \pm 9.5$ days, with $24.6 \%$ in hospital mortality versus $11.2 \% \mathrm{AC},(\mathrm{p}<0.001)$. Clinical 90 day follow up mRS was reported in $64.2 \%$ of cases with independent $\mathrm{mRS} \leq 2$ achieved in $40.4 \%$ versus $37.3 \% \mathrm{AC}, \mathrm{p}=0.31$ ). 90-day mortality was reported in only $0.9 \%$ of cases. Missing data was most common in technical failure, intraprocedural complications, and 90-day mRS amounting to $16.5 \%, 17.3 \%$ and $35.8 \%$ of cases, respectively.
Conclusion Real-world evidence from the NVQI procedural registry suggests posterior circulation stroke to be more common in younger patients with significantly higher NIHSS presentations, and increased utilization of preprocedural MRI for endovascular thrombectomy selection. Given the historical high morbidity and mortality of basilar artery occlusion, there is strong evidence for the benefit of mechanical thrombectomy in the posterior circulation with 3-month functional outcomes nearly identical to thormbectomy outcomes in the anterior circulation.

Disclosures D. Cantrell: None. R. Abdalla: None. A. Shaibani: None. M. Hurley: None. B. Jahromi: None. M. Potts: None. S. Ansari: None.

\section{E-048 PREDICTIVE FACTORS OF GOOD OUTCOMES IN PATIENTS WITH ACUTE BASILAR ARTERY OCCLUSION AFTER ENDOVASCULAR TREATMENT}

S Ha*. Neurosurgery, Chosun univ. Hospital, Gwangju Metropolitan City, Korea, Republic of

10.1136/neurintsurg-2020-SNIS.84

Background and Purpose Basilar artery occlusion (BAO) is associated with high risk of disability and mortality. The objective of this study was to investigate prognostic factors in patients with acute basilar artery occlusion treated with mechanical thrombectomy, focusing on collateral status and recanalization time from symptom onset.

Methods All eligible patients from January 2012 to October 2019 undergoing endovascular treatment (EVT) due to acute BAO were retrospectively reviewed. The baseline posterior circulation collateral status was assessed with Basilar Artery on Computed Tomography Angiography (BATMAN) score and Posterior Circulation Collateral Score (PC-CS). Good outcome was defined as a modified Rankin scale score of $\leq 2$ at 3 months and successful recanalization as thrombolysis in cerebral infarction (TICI) grade $2 \mathrm{~b}$ or 3 . The association between baseline and clinical parameters including and favorable outcome was evaluated with logistic regression.

Results Our sample included a total of 81 eligible patients (49 males, mean age 70.3 years) with a median baseline and discharge National Institute of Health Stroke Scale (NIHSS) score of 12. Patients with good clinical outcome showed a lower baseline National Institute of Health Stroke Scale (NIHSS) score, a more proportion of distal BAO, and a higher BATMAM and PC-CS $(\mathrm{p}<0.001)$. According to the analysis subdivided of patients within and over 6 or 12 hours, the time from symptom onset to recanalization was no correlation with good outcome. Multivariable logistic analysis showed Baseline NIHSS $<15$ (odds ratio [OR] 8.49 95\% confidence interval [CI]: 2.01-35.82, $P=0.004$ ), PC-CS $\geq 6$ (OR 3.79 95\% CI: 1.05-13.66, $P=0.042$ ) and distal BAO (OR 3.67 95\% CI: $1.10-12.26, P=0.035)$ were independent predictors of good clinical outcomes.

Conclusion This study suggested that good collateral circulation and distal BAO are independent predictors of clinical outcome after EVT in patients with acute BAO. In particular, patients with good initial collateral status and distal BAO may consider EVT even if the treatment is started beyond appropriate time line.

Computed tomographic angiography, acute ischemic stroke, basilar artery occlusion, collateral circulation

Disclosures S. Ha: None. 